\title{
Superior Performance of Blind Compared With Sighted Individuals on Bimanual Estimations of Object Size
}

\author{
Melissa Smith, Elizabeth A. Franz, Susan M. Joy, and Kirsty Whitehead
}

University of Otago, Dunedin, New Zealand

\begin{abstract}
Five preliminary experiments on sighted individuals revealed marked overestimation on an object size-estimation task using a bimanual response. These experiments ruled out the possibility that overestimation was due to the mode of visual presentation (whether twodimensional or three-dimensional), the input modality (visual or kinesthetic), or the influence of other visual cues. The main experiment then investigated whether these distortions are due to visual experience by using a variant of the same task to test 24 blind and 24 sighted control participants. Remarkably, the sighted control participants overestimated object size, on average, but the blind participants did not. A follow-up experiment demonstrated that visual memory was the primary influence causing the size overestimations. We conclude that blind individuals are more accurate than sighted individuals in representing the size of familiar objects because they rely on manual representations, which are less influenced by visual experience than are visual memory representations.
\end{abstract}

Close your eyes and imagine a familiar object that you see and manipulate frequently, for example, a loaf of bread. With eyes still closed, indicate the length of the loaf by the distance between your hands. Without moving your hands, open your eyes and observe the distance between your hands. Is this an accurate estimation of the length of the bread you imagined? Our assertion is that, on average, people overestimate size on this type of task because of biases in their memory representations.

Address correspondence to Liz Franz, Action, Brain, and Cognition Laboratory, Department of Psychology, University of Otago, Box 56, Dunedin, New Zealand; e-mail: lfranz@psy.otago.ac.nz.
The closest variants of this task date as far back as the 1950s, when two different laboratories examined size perception using visual or nonvisual judgments. On the basis of the performance of 5 adult subjects on a task involving size judgments of familiar objects, Bolles and Bailey (1956) suggested that perception of size comes not only from visual cues, but also from the past experience that assists one in identifying familiar objects and therefore their approximate sizes. Bartley, Clifford, and Calvin (1955) tested 10 blind children and 10 control participants on a task involving kinesthetic discrimination of the sizes of objects presented in pairs. Their findings were complicated and mixed, but suggested that the visual and kinesthetic modalities might result in size judgments that are biased in different directions, so that in sighted individuals, the two biases cancel one another to result in reasonably good size judgments. Both studies, however, used small numbers of subjects and perceptual judgments that did not indicate the precise size of the object representations held in memory.

More recent research has elucidated direct links between perception and representation of the size of objects placed at different distances from the viewer (Kosslyn, 1978; Lockhead \& Evans, 1979). These studies indicate that the same factors that influence visual perception also influence imagery. In addition, psychophysical methods similar to those used by Bartley et al. (1955) have shown some differences in the functions that relate actual object size to memory-based size judgments and perceptually based size judgments (Moyer, Bradley, Sorenson, Whiting, \& Mansfield, 1978).

We were interested in directly measuring people's estimates of object size in order to investigate internal representations. In a series of five preliminary experiments, we examined the representation of object size in neurologically normal, sighted individuals (see results in Table 1). In the first experiment, 20 


\section{TABLE 1}

Mean Ratios and Standard Errors for the Preliminary Experiments

\begin{tabular}{lccccc}
\hline \hline & \multicolumn{5}{c}{ Experiment } \\
\cline { 2 - 6 } Measure & 1 (visual_objects) & 2 (visual_pictures) & 3 (haptic) & $\begin{array}{c}4 \text { (visual_objects, } \\
\text { response with LEDs) }\end{array}$ & $\begin{array}{c}5 \text { (visual—objects, } \\
\text { response with solid object) }\end{array}$ \\
\hline$M$ & 1.20 & 1.18 & 1.21 & 1.19 & 1.10 \\
$S E$ & 0.025 & 0.031 & 0.033 & 0.028 & 0.040 \\
\hline \hline
\end{tabular}

Note. As indicated, the experiments varied in whether the objects were presented visually (as three-dimensional objects or twodimensional pictures) or haptically. In the first through fourth experiments, subjects estimated size by their hand span; they were blindfolded in the first three experiments but in the fourth were in a dark room and could see LEDs on their fingertips. In the fifth experiment, subjects were blindfolded while estimating size using a block of wood. The ratios reported were calculated by dividing subjects' estimates by the corresponding actual measures of the objects and averaging across objects and subjects. All $t$ tests assessing deviations of mean ratios from 1.00 were significant, including those for size estimates on the initial trial of each object (all $p s<.05)$.

undergraduate volunteers were shown 10 familiar grocery items, each viewed separately from $30 \mathrm{~cm}$ away for $6 \mathrm{~s}$. Participants were then blindfolded and were asked to demonstrate the size of each object when its name was called out in random order. We observed a marked overestimation of object size in approximately $70 \%$ of the individuals' estimates.

In a second experiment, 20 naive undergraduates were presented with the same objects in two-dimensional form-each as a picture on an index card. All the pictures were the same size. Using the same procedures as in the first experiment, participants overestimated object size to the same degree as participants in the first experiment.

In a third experiment, a new group of 20 undergraduates were each blindfolded as they entered our laboratory. They were then presented the same three-dimensional objects as in the first experiment, and were asked to touch them with both hands and identify them. Using the same estimation procedures as before, participants overestimated the size of the objects to the same degree as in the earlier experiments using visual presentation.

To examine whether overestimation might occur because people are unaware of where their hands are in space (given that the size estimations in the previous experiments were performed under blindfolded conditions), in a fourth experiment we placed small (0.5-cm diameter) light-emitting diodes on the tips of the index fingers of the participants' hands. We then presented the objects in the same manner as in the visual-presentation experiments and conducted the size estimation in a dark booth (without blindfolding the participants), so that no visual information was available other than the lights on the fingers. Participants overestimated object size to the same extent as in the previous experiments, despite being able to see the distance between their hands.

A fifth experiment examined whether the participants' overestimation might be related to responding in unfilled space. Specifically, the perception of space might be different when one produces a distance between two endpoints (in this case, the hands) than when one marks a distance on a solid object contained in that space. We assembled an apparatus that consisted of a piece of wood $(2$ in. $\times 4$ in. $\times 4 \mathrm{ft})$ with a cover on each end that could be adjusted to make the exposed piece of wood shorter or longer. Using this apparatus to respond, participants again overestimated object size following visual presentation of threedimensional objects, even with full vision of their estimates. We therefore concluded that the bimanual object-overestimation effect was not due to the mode of visual presentation (whether two-dimensional or three-dimensional), the input modality (visual or kinesthetic), or the influence of other visual cues.

From these preliminary experiments, it was clear to us that sighted individuals, when asked to perform object estimations from memory, tend to overestimate the size of objects that are frequently seen and manipulated in the space around the body (peripersonal space). We were curious whether this effect is due, at least in part, to frequent visual experience with the world. Specifically, sighted individuals see objects every day in different orientations, from different distances, and in the context of a variety of everyday uses. Moreover, somehow a memorial representation of these objects is maintained, evidenced by the ability to readily imagine them. In contrast, people who have been blind for some portion of their recent life experience (or all of their lives) would have had to rely on manual rather than visual representations. It might be that without visual experience, memorial representations are more accurate. Note that it could also be argued that the blind often reach for remembered objects without immediate guidance from a distal sense, and they may therefore have developed good manual size estimates.

Another possibility is that the overestimations of object size in sighted individuals are due not to visual experience, but rather to general properties of the manual response system. For example, studies on unimanual grasping have shown that just prior to reaching an object, the grasp aperture is larger than the object itself (Jeannerod, 1981). Similar properties tend to describe bimanual and unimanual grasping (Tresilian \& Stelmach, 1997). In some ways, producing a grasp aperture that is larger than the object to be grasped might be a mechanism to ensure that a successful grasp eventually occurs, because the aperture becomes smaller as the object is approached. Although our participants were asked not to grasp objects but only to demonstrate their sizes, this task itself might elicit a representation of grasp aperture. By 
this account, we would expect sighted and blind individuals to demonstrate the same degree of size overestimation on our task, given that there is no reason to suspect that the manual response systems operate any differently in the two groups.

\section{METHOD}

\section{Participants}

Twenty-four blind adults recruited from the Royal New Zealand Foundation for the Blind volunteered to be participants after informed consent was obtained in accordance with ethical procedures of the University of Otago. Of these participants, 9 were classified as congenitally blind, having lost their vision within the first 3 years of life (Thinus-Blanc \& Gaunet, 1997). The remaining participants were classified as adventitiously blind. A control subject was matched to each blind subject for sex, education, and age (to within 2 years). None of the control subjects had any known neurological disorder, and all had normal or corrected vision.

\section{Apparatus}

A millimeter tape was used to measure the distance between the participants' hands. A blindfold was used to block vision. The objects whose sizes were estimated were common grocery items, categorized as either vertically or horizontally oriented on the basis of whether the horizontal or vertical dimension was larger in the object's commonly observed orientation. The five vertical objects, ranging in height from $62 \mathrm{~mm}$ to $345 \mathrm{~mm}$, were a 2-L jug of milk, 750-g box of crackers, standard can of vegetables, standard can of soda, and 150-g container of yogurt. The five horizontal objects, ranging in length from $119 \mathrm{~mm}$ to $300 \mathrm{~mm}$ on their longest side, were a standard loaf of bread, carton of a dozen eggs, 500-g package of long spaghetti, 500-g container of butter, and standard candy bar. No interesting effects differentiated the objects within each set; therefore, data were collapsed across objects.

\section{Procedure and Design}

Participants (both sighted and blind) were blindfolded throughout the procedure and were instructed to manipulate each of 10 objects for $6 \mathrm{~s}$ using both hands. To ensure that all items were familiar, we asked participants to report the identity of each object and how often they purchased and used it. If the item was not familiar to the subject, it was replaced with another that was (e.g., a package of spaghetti was replaced with a block of cheese). Such replacement occurred for 6 objects in total, across all subjects (and all analyses indicated that the replacement was inconsequential to the overall effects). After all 10 objects were presented (in random order across subjects), the measurement phase began.

During the measurement phase, the experimenter called out an object name at random from the group of possible objects.
The participant then bimanually estimated the dominant dimension of the object by indicating a hand span, holding the hands in a rigid posture extending from the bent elbows. The distance between the index fingers of the participant's outstretched hands was measured in millimeters. These procedures were repeated until each object was tested 10 times. Between trials, participants were asked to place their hands on their laps, but no feedback was given. For a subsample of the subjects, responses on all trials were also recorded using videotape and then measured off-line. The measurements from the videotapes (adjusted for viewing distance) correlated with the actual measurements from the experimenter with $90 \%$ reliability.

\section{Data Reduction}

A check was first made to ensure that estimates of object size were proportional to actual object size. We measured the length of each horizontal object and the height of each vertical object to produce 10 standard measures. We then computed a correlation coefficient between estimated and actual object size across all trials for each participant. For the blind group, these values ranged from .78 to $.99(M=.92)$, and for the control group, the range was .89 to $.98(M=.94)$. We then transformed the correlation coefficients to $Z$ scores using a Fisher transform and conducted an independent $t$ test to assess any group differences in the transformed correlations. The $t$ test was not significant, $t(46)=0.44, p>.05$.

We divided each recorded measure from the raw data by its corresponding standard measure to produce a ratio for each trial. A ratio of 1 indicated that the size estimate was exactly the same as the standard. A number larger than 1 indicated an overestimation, and a number smaller than 1 indicated an underestimation.

Two analyses were performed on the ratios. First, one-way $t$ tests assessed whether the ratios were significantly different from 1 (i.e., whether participants' estimates were under or over the real object size). Second, we conducted mixed-effects analyses of variance with the between-subjects factor of group (blind, sighted) and the within-subjects factor of orientation (vertical, horizontal).

\section{RESULTS AND DISCUSSION}

The blind group's estimates of object size were not different from the standard measures, $t(23)=1.008, p=.32$. In contrast, the sighted individuals produced overestimations that were significantly larger than the standard, $t(23)=8.52, p<.001$. The means and standard errors of the ratios for the two groups are shown in Figure 1. A highly significant effect of group was found, $F(1,46)=10.60, p=.002$. Note that the two groups also differed on the first trial of each object, so carryover effects of repetition were not responsible for the group differences, $t(46)=-2.60, p=.013$, Cohen's $d=0.75$. These findings 


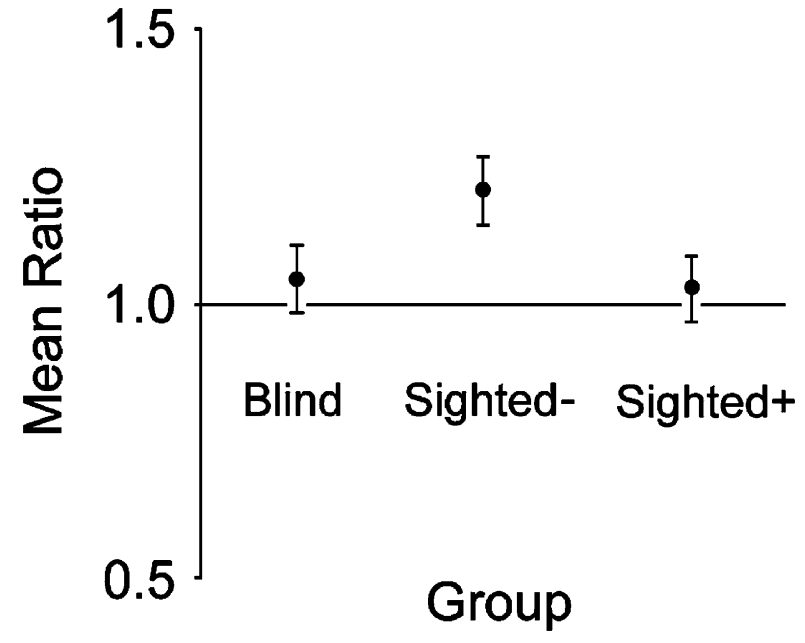

Fig. 1. Means and standard errors of the ratios for the blind and sighted ("Sighted-") groups in the main experiment and the sighted ("Sighted+") group in the follow-up experiment. In the follow-up experiment, subjects could view the object while estimating its size, which removed the visual memory requirement.

indicate that compared with the sighted control group, the blind group produced estimations that were closer, on average, to the actual sizes of objects.

At the end of the experiment, each participant was queried about any strategies he or she used. Interestingly, $50 \%$ of the blind group used a strategy of imagining holding the object. The most frequently reported strategy among the sighted was to imagine the object's size in relation to the size of a body part (see Table 2).

We reasoned that the group differences would be more compelling if at least some aspect of the task was performed similarly across the two groups. For example, performance might be better for horizontally than for vertically aligned objects because the mirror-image symmetry of the body would allow for better distance judgments in the horizontal than in the vertical orientation. If so, this should be the case for both groups. This prediction was supported, with overestimation being lower overall for horizontal objects (mean ratio $=1.08$ ) than for vertical objects (mean ratio $=1.18$ ) $F(1,46)=17.31, p<.001$;

TABLE 2

\section{Strategies Used by Each Group}

\begin{tabular}{lc}
\hline \hline Group and strategy & Percentage of subjects \\
\hline Blind & 50 \\
Imagine holding the object & $29^{\mathrm{a}}$ \\
Try visualizing the object & 13 \\
Imagine body parts as a comparison standard & 8 \\
No particular strategy used & \\
Sighted & 13 \\
Imagine holding the object & 8 \\
Try visualizing the object & 38 \\
Imagine body parts as a comparison standard & 41 \\
No particular strategy used & \\
\hline \hline
\end{tabular}

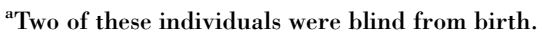

orientation did not interact with group, $F(1,46)=1.152, p=$ .29 . This result suggests that the structural symmetry of the bimanual system benefited the two groups to a similar degree.

We tested an additional 20 (naive) sighted control subjects on a version of the task that allowed them to see the objects but not their hands during the size estimations. On each trial, the object was placed directly in front of the subject on a small shelf (30 cm away) that obstructed the subject's view of her or his hands while keeping the object in clear view. We then conducted the size-estimation procedures. Under these conditions, there was no tendency to overestimate $(p>.05)$. Thus, when we removed the visual memory requirement by allowing sighted participants to demonstrate size with each object in full vision, the tendency to overestimate was significantly reduced.

In summary, these findings support our novel hypothesis that the memory representations of sighted individuals overestimate object size unless visual memory demands are relaxed. The memory representations of people who have lost vision for some portion of their lives are less likely to overestimate object size, suggesting that blind people may have developed accurate haptic size representations in support of reaching and grasping actions.

Acknowledgments-This research was supported by Otago Research Grant MFUB26 to Liz Franz. We are grateful to the Royal New Zealand Foundation for the Blind for their invaluable assistance in recruiting participants, the participants for their generous contributions of time and effort, and John Kennedy and an anonymous reviewer for very instructive suggestions during the review process.

\section{REFERENCES}

Bartley, S.H., Clifford, L.T., \& Calvin, A.D. (1955). Effect of visual imagery on tactual and kinesthetic space perception. Perceptual and Motor Skills, 5, 177-184.

Bolles, R.C., \& Bailey, D.E. (1956). Importance of object recognition in size constancy. Journal of Experimental Psychology, 51, 222-225.

Jeannerod, M. (1981). Intersegmental coordination during reaching at natural visual objects. In J. Long \& J. Baddeley (Eds.), Attention and performance IX (pp. 153-169). Hillsdale, NJ: Erlbaum.

Kosslyn, S. (1978). Measuring the visual angle of the mind's eye. Cognitive Psychology, 10, 356-389.

Lockhead, G., \& Evans, N. (1979). Emmert's imaginal law. Bulletin of the Psychonomic Society, 13(2), 114-116.

Moyer, R., Bradley, D., Sorenson, M., Whiting, J., \& Mansfield, D. (1978). Psychophysical functions for perceived and remembered size. Science, 200, 330-332.

Thinus-Blanc, C., \& Gaunet, F. (1997). Representation of space in blind persons: Vision as a spatial sense? Psychological Bulletin, 121(1), 20-42.

Tresilian, J., \& Stelmach, G. (1997). Common organization for unimanual and bimanual reach-to-grasp tasks. Experimental Brain Research, 115, 283-299.

(RECEIVED 10/28/03; REVISION ACCEPTED 12/16/03) 